\title{
DNA Vaccine Targeting Gonadotropin-Releasing Hormone Receptor and Its Application in Animal Contraception
}

\author{
Alexandre Samoylov ${ }^{1} \cdot$ India Napier $^{1} \cdot$ Nancy Morrison $^{1} \cdot$ Anna Cochran $^{1} \cdot$ Bettina Schemera $^{2} \cdot$ James Wright $^{3}$. \\ Russell Cattley ${ }^{3} \cdot$ Tatiana Samoylova $^{1,3}$
}

Published online: 17 November 2018

(c) The Author(s) 2018

\begin{abstract}
Overpopulation of free-roaming and wildlife animals negatively affects economy and public health in many parts of the world. Contraceptive vaccines are viewed as a valuable option for reducing numbers of unwanted animals. This study develops vaccines for potential use in animal contraception exploiting a DNA platform. Objectives of the study were to generate DNA constructs directed against gonadotropin-releasing hormone receptor (GnRHR), a crucial molecular player in animal reproduction, and characterize them for ability to promote immune responses and suppression of reproductive parameters in vivo. DNA constructs were created to encode for a recombinant protein composed of two domains: GnRHR, the target antigen, and ubiquitin (Ub), a support protein. Ub-GnRHR constructs administered intramuscularly or intradermally or containing different promoters were compared. CMV and EF1 $\alpha$ promoters were shown to be superior to CAG. In fertility trials, mice immunized intradermally with Ub-GnRHR construct driven by EF1 $\alpha$ had a significantly lower number of fetuses. Importantly, the impaired fertility was achieved with a single DNA immunization and without the use of adjuvants. The study demonstrated for the first time that targeting the GnRH receptor with DNA-based vaccines could be a viable option for animal contraception.
\end{abstract}

Keywords DNA vaccine $\cdot$ Gonadotropin-releasing hormone $\cdot$ Immunocontraception

\section{Introduction}

Expanding populations of free-ranging and wildlife animals, including cats, dogs, deer, wild pigs, and others, affect the economy and public health in many parts of the world. The World Health Organization, for example, estimated that there are 200 million stray dogs worldwide [1]. These dogs often carry infections (e.g., deadly rabies virus) and parasites transmissible to humans and domestic animals [2]. They also are capable of attacking people, poultry, livestock, and pets [3]. Feral cats also are reservoirs for various

Tatiana Samoylova

samoiti@auburn.edu

1 Scott-Ritchey Research Center, College of Veterinary Medicine, Auburn University, 1265 HC Morgan Drive, Auburn, AL 36849, USA

2 Laboratory Animal Health, College of Veterinary Medicine, Auburn University, Auburn, AL, USA

3 Department of Pathobiology, College of Veterinary Medicine, Auburn University, Auburn, AL, USA zoonotic diseases and parasites [4, 5] and, in addition, play a significant role in negative changes to the environment and native wildlife habitats killing a large number of birds, small mammals, and reptiles [6]. On the other hand, feral cats and free-roaming dogs suffer serious diseases, car accidents, cruelty, and starvation. Fertility management using contraceptive vaccines for cats and dogs as well as for some wildlife species (e.g., deer, horses, elephants) has been recognized to be among the most promising and humane approaches in reducing their numbers [7-11].

DNA vaccines are composed of a bacterial plasmid that is engineered to encode an antigen of interest under control of a strong promoter for high-level and long-lasting protein expression in mammalian cells. One of the greatest advantages of DNA-based vaccines is their ability to activate both humoral and cell-mediated arms of the immune system, making them attractive vaccine vehicles [12]. DNA vaccines are cost-effective since recombinant plasmids can be produced at large scale in bacteria and purified by simple methods. They are more temperature stable compared to protein vaccines, allowing for storage, transport, and use 
in variable field conditions. Importantly, DNA vaccines are molecularly defined and may provide long-term persistent immune responses specific to the antigen of interest. DNA plasmids themselves intrinsically include $\mathrm{CpG}$ immunostimulatory sequences that may act as potent adjuvants. Numerous studies in animals as well as dozens of human clinical trials have provided firm evidence that DNA vaccines are safe [12-14]. Several DNA vaccines for veterinary applications have been licensed, including West Nile virus vaccine for horses (West Nile-Innovator ${ }^{\circledR}$, Fort Dodge Animal Health, Fort Dodge, IA, USA) and anti-melanoma vaccine for dogs (Oncept ${ }^{\mathrm{TM}}$, Merial, Lyon, France).

The DNA platform has been utilized for development of anti-fertility vaccines against several reproductive targets, including zona pellucida [15-18], fertilin B subunit [19], YLP12 [20], fertilization antigen FA-1 [21], GnRH peptide [22, 23], testis-specific sodium-hydrogen exchanger protein [24], and cation channel sperm-associated protein 1 [25]. Gonadotropin-releasing hormone receptor (GnRHR) is an unexplored target in this respect. This is a receptor for a hypothalamic decapeptide GnRH that specifically binds and activates GnRHR on gonadotrope cells in the pituitary gland. The receptor regulates synthesis and secretion of pituitary gonadotropins that are essential for reproductive functions, including gametogenesis and synthesis of gonadal steroids [26]. The GnRH receptor is an attractive target for animal contraception for several reasons. First, GnRHR is a vital link in hypothalamus-pituitary-gonadal cascade of reproductive events disruption of which leads to infertility. Second, the location of the pituitary gland outside of the brain facilitates systemic access to GnRHR without crossing the blood brain barrier, unlike delivery to the hypothalamic contraceptive targets. Third, disruption of GnRH-GnRHR interaction stops reproduction and sexual behavior in both male and female animals.

The objectives of this study were to generate several DNA constructs directed against GnRHR which are composed of different domains and to characterize these constructs for their ability to promote specific immune responses and suppression of reproductive parameters in vivo. Such DNA-based vaccines have potential applications in animal contraception.

\section{Materials and Methods}

\section{Animal Samples and Generation of Ub-fGnRHR Constructs}

All animal samples were collected under protocols approved by Auburn University Institutional Animal Care and Use Committee (AU IACUC). For molecular cloning of feline GnRHR (fGnRHR), pituitary glands were obtained from three normal cats (6-month-old male, 11-month-old female, and 3-year-old female). For molecular cloning of ubiquitin, whole brains were collected from CD-1 male mice. RNAs were isolated from collected tissues using RNeasy Lipid Tissue Mini Kit (Qiagen, Germantown, MD, USA) as directed by the manufacturer.

To generate the Ub-fGnRHR construct, two support plasmids were produced: (1) fGnRHR plasmid encoding for feline GnRHR gene and (2) Ub plasmid encoding for murine ubiquitin gene. To produce the fGnRHR plasmid, fGnRHR gene was amplified from total RNA (isolated from cat pituitary) with gene-specific primers (A) and (B) (Table 1) and the amplified product was cloned into EcoRI and $\mathrm{XbaI}$ restriction sites of pcDNA ${ }^{\mathrm{TM}} 3.1^{(+)}$Mammalian Expression Vector (Invitrogen, Carlsbad, CA, USA). To produce the Ub plasmid, ubiquitin gene was amplified from total RNA (isolated from mouse brain tissue) with gene-specific primers (C) and (D) (Table 1) and the amplified product was cloned into EcoRI and NotI restriction sites of pcDNA3.1 plasmid (Invitrogen). The final Ub-fGnRHR plasmid was created in two steps. First, fGnRHR sequence was amplified from fGnRHR support plasmid using primers (E) and (F) (Table 1) and cloned into NotI and XbaI restriction sites. Second, the
Table 1 Primer sequences used for cloning and amplification of murine ubiquitin and feline GnRHR genes

\begin{tabular}{ll}
\hline Gene/construct name & Primer name/nucleotide sequence (5'-3') \\
\hline fGnRHR & A: ACTCgAATTCgCCACCATggCAAgTgCCCCTCC \\
& B: ACTCTCTAgATTACAgAgAgAAATACCCA \\
Ub & C: CgACTCgAATTCgCCACCATgCAgATCTTCgTgAAg \\
& D: gACTATgCggCCgCCACCTCTCAggCgAAggACC \\
Eb-fGnRHR & E: TCTTTATgCggCCgCATggCAAgTgCCCCTCCTg \\
& F: ACTCTCTAgACTAgTggTgATggTgATgATgCAgAgA- \\
& gAAATACCCATA \\
& G: ATACTCggATCCgCCACCATgCAgATCTTCgTgAAg \\
& H: gACTATgCggCCgCTCgCACCTCTCAggCgAAggACCAg \\
& I: ATACTCggTACCgCCACCATgCAgATCTTCg \\
& J: ACTCTCTAgACCTACAgAgAgAAATACCCATATATAAg \\
\end{tabular}


ubiquitin sequence was amplified from Ub support plasmid using primers $(\mathrm{G})$ and $(\mathrm{H})$ (Table 1) and ligated into BamHI and NotI restriction sites of the same plasmid yielding a construct encoding for recombinant Ub-fGnRHR protein.

Three additional constructs encoding Ub-fGnRHR recombinant protein were created using pSF-CAG-Kan (Cat \#OG505), pSF-EF1 Alpha (Cat \#OG43), and pSF-CMVKan (Cat \#OG10) plasmids (Oxford Genetics, Begbroke, UK), which are identical except for the promoters. The UbfGnRHR DNA fragment was amplified using primers (I) and (J) and cloned in Kpn1 and Xba1 restriction sites of the corresponding plasmids. All primers were purchased from TIB MolBiol (Adelphia, NJ, USA).

One-Step RT-PCR Kit (Qiagen) was used for reverse transcription and amplification of total RNA. DNA products were purified using a QIAquick PCR Purification Kit (Qiagen). T4 DNA ligase and all restriction enzymes were purchased from New England BioLabs (Ipswich, MA, USA). Sequencing of PCR products was performed by Massachusetts General Hospital DNA Core Center for Computational and Integrative Biology (Cambridge, MA).

\section{Evaluation of mRNA Expression}

For transfection, Chinese hamster ovary (CHO-K1) cells (CCL-61, ATCC, Manassas, VA, USA) (aliquots of $5 \times 10^{5}$ cells) were mixed with $1.5 \mu \mathrm{g}$ plasmid DNA in a $100 \mu \mathrm{l}$ volume. Electroporation was performed at $250 \mathrm{~V}, 30 \mathrm{msec}$ pulse length. The transfected cells were cultured for $48 \mathrm{~h}$ and total RNA isolated. To confirm transcriptional expression, Ub-fGnRHR mRNA was amplified with standard primers T7 and BGH and subjected to $0.8 \%$ agarose electrophoreses.

\section{Immunization of Mice}

All animal procedures were performed according to protocols approved by AU IACUC. Mice were 8-week-old CD-1 (outbred) males purchased from Charles River Laboratories (Willington, MA, USA). To conduct experiments with different DNA administration routes, mice (eight/group) were randomized into three treatment groups: (1) pcDNA(CMV)UbfGnRHR plasmid (see Fig. 1 for design of all plasmids and their abbreviations) administered intramuscularly
Fig. 1 Construction and expression of DNA plasmids with the Ub-fGnRHR insert. a Schematic diagram of the constructs and their abbreviated names. b RT-PCR detection of UbfGnRHR mRNA in CHO-K1 cells. Lanes: (1) UbfGnRHRtransfected cells, (2) UbfGnRHR-transfected cells, no RT control, (3) not transfected cells (negative control), (4) plasmid containing UbfGnRHR insert (positive control), (5) $1 \mathrm{~Kb} \mathrm{MW}$ ladder. The expected molecular weight (1366 bp) band for murine ubiquitin-feline GnRHR PCR product is shown by arrow

\section{A}

pcDNA3.1 plasmid backbone (Invitrogen)

pcDNA(CMV)-UbfGnRHR

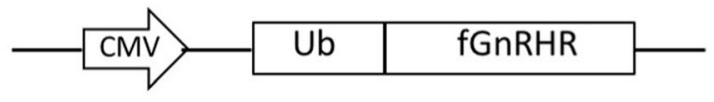

pSF plasmid backbone (Oxford Genetics)

pSF(CMV)-UbfGnRHR

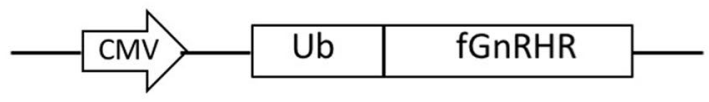

pSF(EF1 $\alpha)$-UbfGnRHR

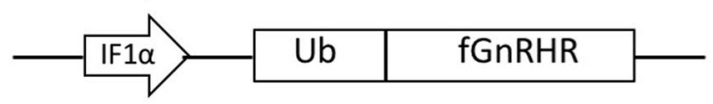

pSF(CAG)-UbfGnRHR

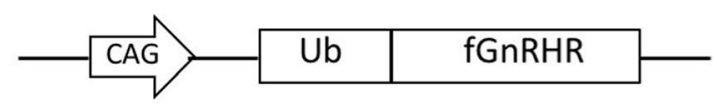

\section{B}

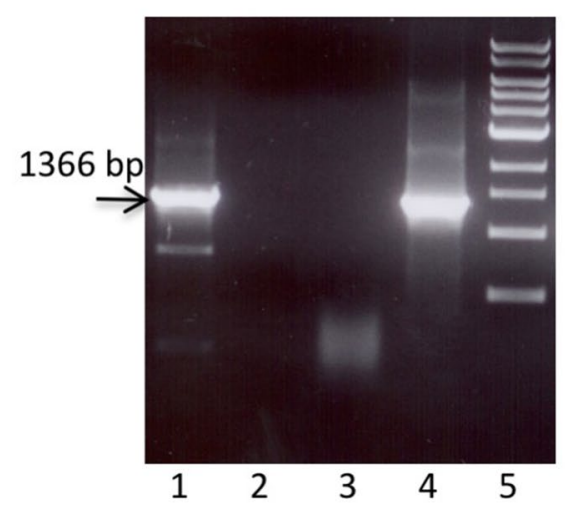


(IM), (2) pcDNA(CMV)-UbfGnRHR plasmid immobilized on $200 \mathrm{~nm}$ chitosan-coated PLGA nanoparticles (Degra$\operatorname{dex}^{\mathrm{TM}}$ PLGA Nanospheres, Phosphorex, Inc, Hopkinton, MA, USA) administered IM, and (3) pcDNA(CMV)-UbfGnRHR plasmid administered intradermally (ID). In both IM groups, each animal was immunized with $100 \mu \mathrm{g}$ DNA (50 $\mu \mathrm{g}$ DNA in $50 \mu \mathrm{l}$ PBS per quadriceps femoris muscle). In the ID group, mice were first anesthetized via intraperitoneal injection of ketamine/dexmedetomidine, and then, $15 \mu \mathrm{g}$ DNA was injected into the dermis of each ear pinna (30 $\mu \mathrm{g}$ DNA per animal, $30 \mu \mathrm{l}$ volume/injection). The experiment on optimization of the delivery route continued 12 weeks. To conduct experiments testing different promoters, mice (eight/group) were randomized into the following groups: (1) treated with pSF(CMV)-UbfGnRHR plasmid, (2) treated with $\operatorname{pSF}(\mathrm{EF} 1 \alpha)-\mathrm{UbfG}$ RHR plasmid, and (3) treated with pSF(CAG)-UbfGnRHR plasmid. In this experiment, all mice were immunized ID as described above. The experiment on promoter optimization continued 25 weeks. Mouse testes were harvested at necropsy, weighted, and fixed in $4 \%$ paraformaldehyde, $0.1 \mathrm{M}$ phosphate buffer. The tissues were embedded in paraffin. Tissue sections were stained using Hematoxylin and Eosin standard protocol.

\section{ELISAs: Detection of GnRHR Antibodies and Testosterone}

GnRHR antibodies were detected by ELISA using a synthetic 29 amino acid peptide (AnaSpec, Fremont, CA, USA) representing $\mathrm{N}$-terminal extracellular domain of the feline GnRH receptor protein (MASAPPEQNQNHCSAINNSIPLMQGNLP-K-Biotin) to serve as the fGnRHR antibody detector molecule. The step-by-step protocol was described previously [27]. To measure serum testosterone, a Mouse and Rat Testosterone ELISA kit (ALPCO, Salem, NH, USA) was used as directed by the manufacturer. The absorbance was measured at $490 \mathrm{~nm}$ using Synergy HT reader (BioTek, Winooski, VT, USA).

\section{RT qPCR}

The pituitary glands were collected at necropsy and total RNA isolated. One-step RT qPCR was performed with iTAq Universal Probes One-Step Kit (Bio-Rad Laboratories, Inc., Hercules, CA, USA) according to the manufacturer's protocol using CFX96 Real-Time PCR Detection System (Bio-Rad). Two sets of primers and probes were designed. For murine GnRHR, forward primer was TCTgCAATgCCAAAATCA TCT, reverse primer was TAgCgAATgCgACTgTCATC, and probe was 6FAM-AgCgTTCTCAgCCgAgCTCTTgg-BHQ1. For murine beta actin (housekeeping gene), forward primer was ATTACTgCTCTggCTCCTAgC, reverse primer was
gggCCggACTCATCgTA, and probe was 6FAM-TCCACA TCTgCTggAAggTggACA-BHQ1.

\section{Fertility Trials}

Fertility trials were conducted with three test and one control groups of mice (eight mice per group). In the treatment groups, mice were immunized with $\mathrm{pSF}(\mathrm{CMV})-\mathrm{UbfGnRHR}$, pSF(EF1 $\alpha)$-UbfGnRHR, or pSF(CAG)-UbfGnRHR plasmid using the intradermal protocol as described in the mouse immunization section above. Twenty-two weeks post treatment, male mice were paired for mating with normal (not treated) female mice at 1:1 ratio and housed one pair per cage. In the control group, normal males were paired with normal females as for the treatment groups. Female mice were checked daily for vaginal plugs (indication of mating). Starting 1 week after pairing, female mice were checked daily for signs of pregnancy (enlarged abdomen and presence of fetuses detected by palpation). Male and female mice were separated after 2 weeks of pairing. Pregnant female mice were euthanized at the time when the pregnancy was confirmed and the number of fetuses was counted.

\section{Data Entry and Analysis}

GnRHR antibody responses in sera of immunized mice were expressed as means of OD490 $\pm \mathrm{SE}$ for individual mouse groups at serum dilution of 1:1600. Testosterone data were entered and stored in Microsoft Excel $2013^{\mathrm{TR}}$ and analyzed using Statistical Analysis System software (SAS, release version 9.4, Cary, NC). The general linear model (GLM) for repeated measures analysis of variance (ANOVA) was used to detect differences in testosterone levels within groups by using reference contrasts comparing each week post-immunization with pre-immunization data. qPCR data were analyzed using GLM for ANOVA and Dunnett's test to compare each of the treated groups to the control. The data obtained in the fertility trials (number of fetuses per female mouse) were analyzed with GLM for ANOVA and Dunnett's test to compare three test groups to the control group (normal males paired with normal females). For the mean testicular size, each group was compared to the control using the GLM for ANOVA and Dunnett's test. Within group antibody values were analyzed using the GLM for repeated measures analysis of variance and reference contrasts comparing each week post-immunization with pre-immunization data. 


\section{Results}

\section{Generation and Expression of Ub-fGnRHR Constructs}

To generate constructs targeting GnRHR, commercially available DNA vectors for mammalian expression were used to incorporate sequences encoding for GnRHR, the target antigen, and ubiquitin (Ub), a support protein. Since GnRHR is a self-protein, an oligonucleotide sequence encoding heterologous antigen (feline GnRHR) was used to overcome self-tolerance in mice. In addition, ubiquitin was utilized for induction of anti-self immunity via targeting recombinant GnRHR protein to the ubiquitin-proteasome system for effective degradation and antigen presentation.

In total, four constructs were generated (Fig. 1a). All of them had Ub-fGnRHR inserts. One of the constructs (named pcDNA(CMV)-UbfGnRHR) was generated using an Invitrogen pcDNA3.1 plasmid containing CMV promoter. This construct was used in mouse experiments that tested different administration routes. Three additional constructs were created using Oxford Genetics pSF plasmids, which have identical backbones, but differ by promoters (CMV, EF1 $\alpha$, or CAG). These constructs named pSF(CMV)-UbfGnRHR, pSF(EF1 $\alpha)$-UbfGnRHR, and pSF(CAG)-UbfGnRHR were used to identify the best promoter for strong expression of the GnRHR antigen leading to suppression of testosterone in immunized mice.

Expression of the encoded antigen was verified in vitro by transient transfection of CHO-K1 cells. Using RTPCR, mRNA specific to the encoded genes was detected in transfected cells indicating successful transcription of the ubiquitin and fGnRHR genes (Fig. 1b). Sequence analysis confirmed that the PCR products had $100 \%$ accurate sequences for the encoded genes. We were not able to demonstrate a clear-cut identification of fGnRHR protein in transfected cells either by using GnRHR antibodies or the inositol phosphate 1 (IP-1) method (P-One Cisbio kit, quantifies G-protein coupled receptors), most likely, due to the fact that antibodies that recognize feline GnRHR reliably are not available, and/or because of low expression of the protein. Indirectly, fGnRHR protein expression was confirmed later in the study via detection of anti-fGnRHR antibodies in fGnRHR-immunized mice.

\section{Ub-fGnRHR Construct: Delivery Optimization in Mice}

Three delivery routes/methods were tested in mice using the pcDNA(CMV)-UbfGnRHR construct: (1) intramuscular injection into quadriceps muscle of the construct alone, (2) intramuscular injection into quadriceps muscle of the construct immobilized on poly(lactic-co-glycolic acid) (PLGA) nanoparticles, and (3) intradermal injection into ear pinna of the construct alone. PLGA was used in the study since it is known to act as an adjuvant and for slow DNA release. The experiment continued for 12 weeks and sera collected from the immunized animals at four time points were assayed for GnRHR antibodies and testosterone.

GnRHR antibodies were not found in sera of mice immunized intramuscularly at any time points. GnRHR antibodies were detected in sera of mice immunized intradermally at week 12 post immunization. Also, mice immunized via intramuscular administration (with or without PLGA particles) did not demonstrate significant suppression of testosterone (Fig. 2a, b) ( $p>0.05)$. In contrast, the mean testosterone value for the intradermal group was significantly lower at week 12 post-immunization as compared to its preimmunization value (Fig. 2c) $(p<0.05)$. Based on GnRHR antibody responses and serum testosterone suppression, intradermal was found to be superior as compared to intramuscular delivery and, therefore, was used as the preferred administration route in experiments that followed.

\section{Ub-fGnRHR Constructs: Promoter Optimization in Mice}

Three DNA constructs encoding Ub-fGnRHR antigen driven by different promoters were tested to identify a promoter that supports the antigen expression resulting in the most prominent and prolonged immune responses and testosterone suppression in mice. All constructs were created using pSF core plasmids with identical bacterial backbones, but with different promoters (CMV, EF1 $\alpha$, or CAG). pSF(CMV)-UbfGnRHR, pSF(EF1 $\alpha)$-UbfGnRHR, or pSF(CAG)-UbfGnRHR constructs were administered in mice once intradermally and blood was collected from the immunized animals eight times during the experiment, which continued for 25 weeks.

GnRHR antibodies were detected in serum samples collected from mice immunized with $\mathrm{pSF}(\mathrm{CMV})$-UbfGnRHR or $\mathrm{pSF}(\mathrm{CAG})-\mathrm{UbfGnRHR}$ constructs starting at week eight (Fig. 3) or with $\operatorname{pSF}(\mathrm{EF} 1 \alpha)$-UbfGnRHR starting at week 12. From that time point, the antibody levels were increasing and persisted in the blood of all three mouse groups until week 22 of the experiment $(p<0.05)$. The antibodies were detectable in the $\operatorname{pSF}(E F 1 \alpha)$-UbfGnRHR mouse group until the end of the experiment (week 25). Significant testosterone suppression was found in mice immunized with $\mathrm{pSF}(\mathrm{CMV})$-UbfGnRHR and $\mathrm{pSF}(\mathrm{EF} 1 \alpha)$-UbfGnRHR constructs as compared to their pre-immunization group values (Fig. 4a, b). In pSF(EF1 $\alpha$ )-UbfGnRHR group, a significant decrease in testosterone was first observed at week 12 and in the pSF(CMV)-UbfGnRHR group at week 16 post 

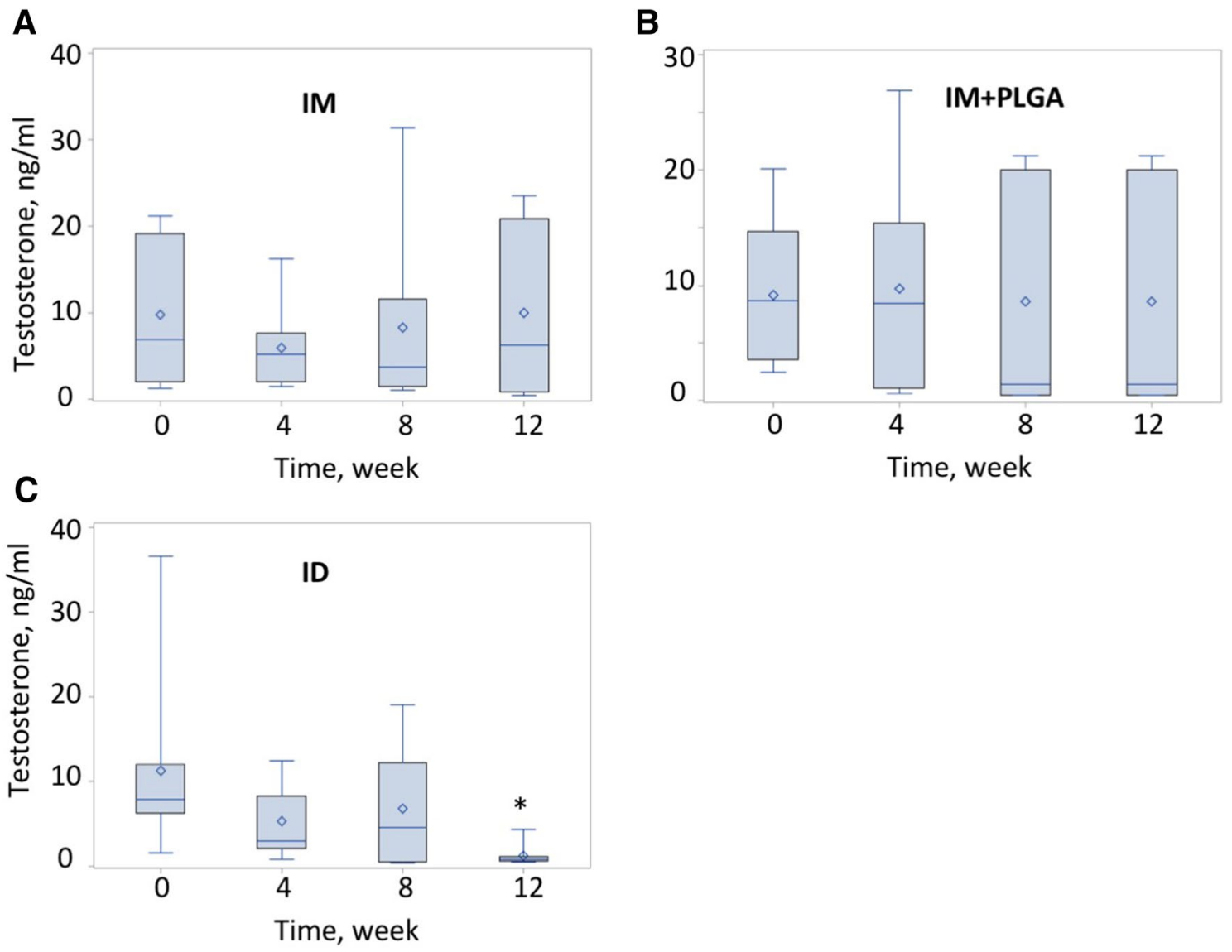

Fig. 2 Serum testosterone in mouse sera prior to and post immunization with the pcDNA-UbfGnRHR construct delivered via different administration routes/methods (distribution by week). a Intramuscular administration. b Intramuscular administration combined with delivery by PLGA nanoparticles. c Intradermal administration into ear pinna. Sera were collected before immunization and at three time

immunization $(p<0.05)$. In both groups, testosterone suppression continued for at least $6-8$ weeks. No statistically significant $(p>0.05)$ decrease in testosterone was observed in mice immunized with the $\mathrm{pSF}(\mathrm{CAG})$-UbfGnRHR construct (Fig. 4c).

To obtain insights on possible mechanisms leading to testosterone suppression, pituitary glands were collected from immunized mice at necropsy and GnRHR mRNA was quantified using qPCR analysis. Statistically significant decrease in GnRHR mRNA expression was found in all treatment groups, as compared to untreated control mice (Fig. 5). Also, the total weight of testes (left plus right) removed at necropsy was measured and the testicular weight expressed as a percentage of the body weight calculated for each group. This parameter for all test groups was lower than for the control group. For mice immunized with pSF(CMV)-UbfGnRHR, pSF(EF1 $\alpha)$-UbfGnRHR, and pSF(CAG)-UbfGnRHR constructs, it was equal to $0.57,0.49$, and $0.56 \%$, respectively, while for the control group it was $0.63 \%$. For

points $(4,8$, and 12 weeks) after immunization and assayed for testosterone content. Box plots: rectangular boxes represent the interquartile range (25th to 75 th percentile); diamonds inside boxes are group mean values; lines inside boxes are group medians; vertical lines (whiskers) represent minimum and maximum values for each group. $P<0.05$ is noted with an asterisk

$\mathrm{pSF}(\mathrm{EF} 1 \alpha)$-UbfGnRHR group, the difference was statistically significant. Histological evaluation of mouse testes demonstrated mild changes in a small number of test animals, including alteration in seminiferous tubules characterized by occasional basally oriented retention of spermatid nuclei (two mice) and formation of sperm granuloma (one mouse) in the pcDNA(CMV)-UbfGnRHR group immunized intradermally.

\section{Fertility Trials in Mice}

Fertility trials in mice were conducted to evaluate whether their fertility was affected by immunizations with DNA UbfGnRHR constructs. Twenty-two weeks post treatment, male mice immunized with pSF(CMV)-UbfGnRHR, pSF(EF1 $\alpha)$ UbfGnRHR, or pSF(CAG)-UbfGnRHR constructs were paired with normal females and the number of fetuses per female were counted. Number of fetuses in the test group treated with UbfGnRHR construct driven by EF1 $\alpha$ promoter 


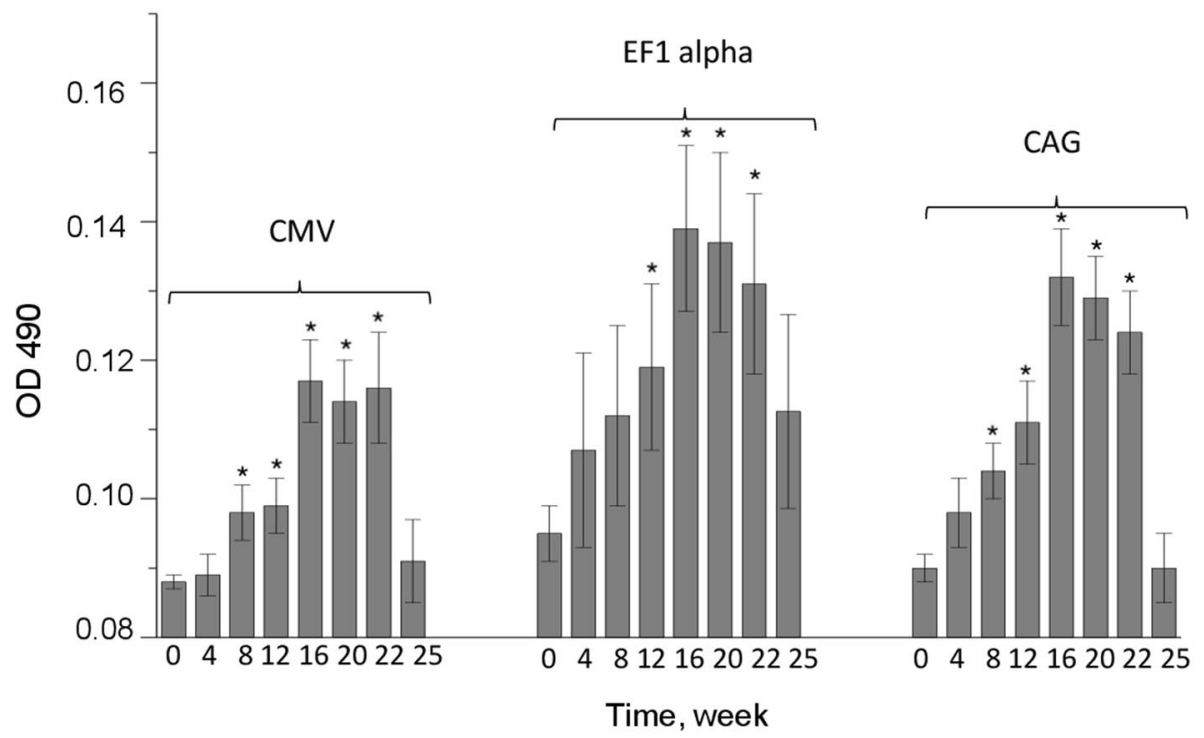

Fig. 3 GnRHR antibodies in mouse sera prior to and post immunization with the pSF-UbfGnRHR constructs driven by different promoters, CMV, EF1 $\alpha$, or CAG. Sera were collected before immunization and at seven time points after immunization and assayed for GnRHR antibodies by ELISA. A twenty-nine amino acid synthetic peptide representing the $\mathrm{N}$-terminal extracellular domain of the feline $\mathrm{GnRH}$

had a significantly lower number of fetuses (group mean 9.25) as compared to untreated control group (14 fetuses), $(p<0.05)$ (Fig. 6). The number of fetuses in the remaining two treatment groups was lower than in the control group, but not significantly $(p>0.05)$.

\section{Discussion}

The focus of this study was on construction of DNA-based vaccines that target the GnRH receptor, a crucial player in animal reproduction. To overcome GnRH receptor tolerance, DNA plasmids were designed to include a heterologous (feline) GnRHR-encoding sequence for immunization of mice. A similar approach was shown to be successful by others. For example, Oncept ${ }^{\mathrm{TM}}$, an effective DNA vaccine for treatment of malignant melanoma in dogs, encodes heterologous human tyrosinase that breaks tolerance and generates substantial immune responses against the canine protein $[28,29]$. In another instance, cats and dogs immunized with bovine luteinizing hormone receptor (LHR) protein produced anti-receptor antibody causing significant suppression of their reproductive functions [30, 31]. Immunizations with follicle stimulating hormone receptor (FSHR) or LHR using human- or porcine-derived proteins elucidated immune reactions in mice that effectively inhibited their fertilization [32-34]. receptor protein was used as the GnRHR antibody detector molecule. The duration of the experiment was 25 weeks. Data are presented for individual mouse groups as OD490 means of two repeats \pm SE at serum dilutions of 1:1600. Significance level of $p<0.05$ is shown with an asterisk

Additionally, to override GnRHR selfness, DNA plasmids in this study were constructed to encode ubiquitin, a small protein that targets intracellular proteins to proteasome. Ubiquitin is a 76 amino acid long highly conserved intracellular protein that has been found in almost all tissues of eukaryotic organisms [35]. Vaccination with plasmid DNA encoding ubiquitin-conjugated protein allows expression of the mono-ubiquitinated antigen in cytoplasm of the transfected antigen presenting cells (APCs). Monoubiquitinated substrate then triggers a cascade of polyubiquitination, targeting the antigen to the proteasome for effective protein degradation to antigenic peptides, which form complexes with MHC-I molecules. Subsequently, MHC-I/ peptide complexes are transported to the surface of APCs and activate antigen-specific $\mathrm{CD} 8^{+} \mathrm{T}$ cells $[36,37]$. Chou et al. [38], for example, constructed a DNA vaccine encoding for ubiquitin fused to aquaporin, a water channel protein highly expressed in the vascular endothelial cells of cancers. Vaccination with the resulting plasmid DNA successfully established cytotoxic T lymphocytes (CTLs) specific to aquaporin, which killed aquaporin-expressing endothelial cells in tumor vasculature, preventing tumor growth. Moreover, there is a report showing that fusion of ubiquitin to a self-antigen (tyrosinase-related protein 2) facilitated formation of specific CTL epitopes, resulting in immunity against cells expressing self-antigen [36].

Various methods for delivery of the DNA-GnRHR construct in mice were tested in the present study. Intramuscular 

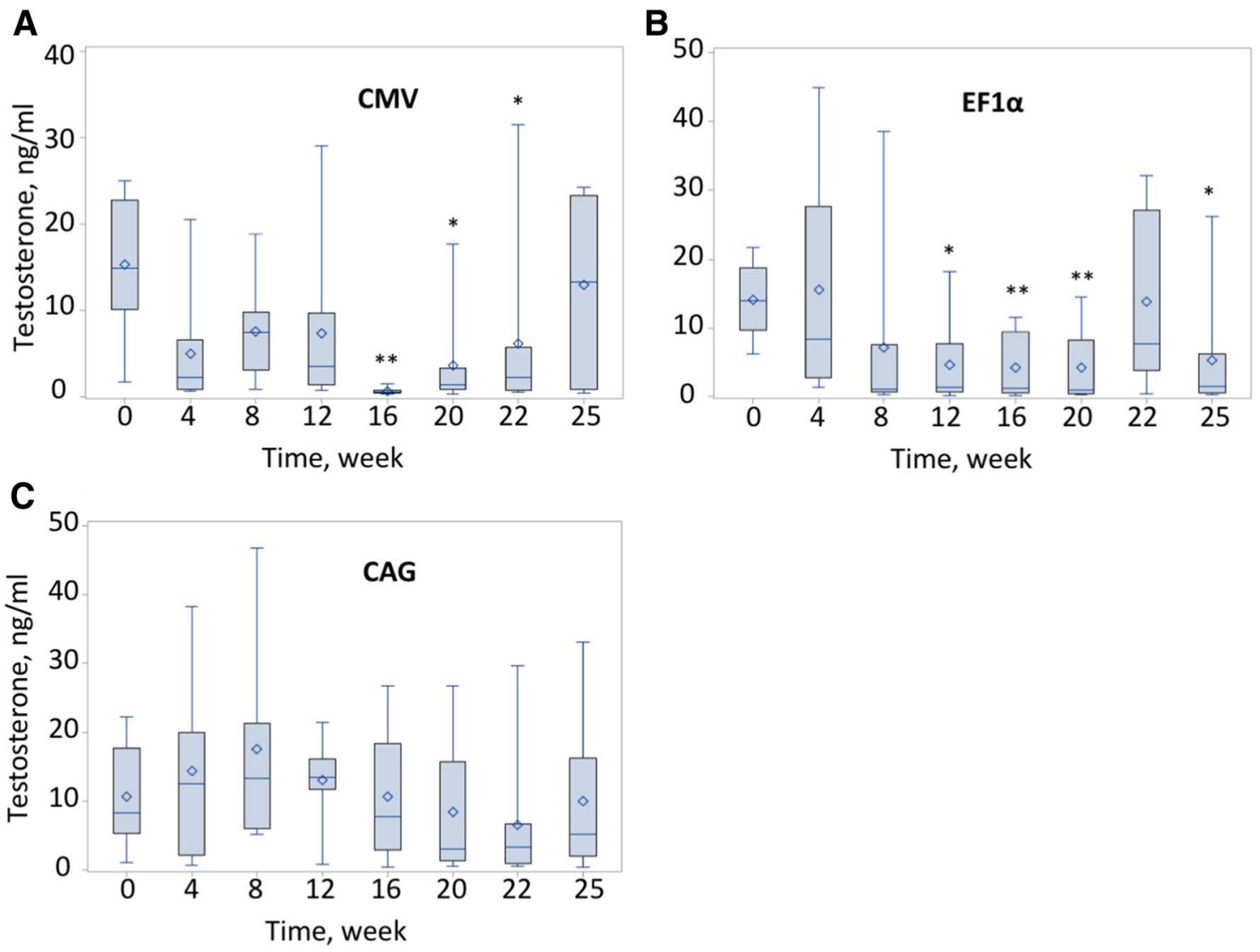

Fig. 4 Testosterone in mouse sera prior to and post immunization with pSF-UbfGnRHR constructs driven by different promoters, CMV, EF1 $\alpha$, or CAG (distribution by week). a Mice immunized with $\mathrm{pSF}(\mathrm{CMV})-\mathrm{UbfGnRHR}$ construct. b Mice immunized with $\operatorname{pSF}(E F 1 \alpha)$-UbfGnRHR construct. c Mice immunized with

pSF(CAG)-UbfGnRHR construct. Serum samples were collected before immunization and at seven time points after immunization and assayed for testosterone. The duration of the experiment was 25 weeks. Box plots presented as in Fig. 2. $P<0.05$ and $p<0.01$ are noted in the figure with one or two asterisks, respectively

delivery of the construct alone and on PLGA particles did not promote immune responses leading to testosterone suppression; however, intradermal administration was effective. Even though muscle was the first and is the most traditional site for DNA injections, skin was shown to be an immunologically better site for DNA immunization due to abundant presence of immune cells such as Langerhans (dendritic cells) [39]. Moreover, intra-ear pinna immunization is a type of administration that was demonstrated to induce superior immune responses to DNA vaccines compared to subcutaneous inoculation [40]. This might be explained by the special structure of the pinna, which contains two layers of epidermis and dermis (separated by ear cartilage) with a high number of dendritic cells within a restricted area connected with major superficial cervical draining lymph nodes. Importantly, efficacy of intra-pinna DNA vaccination was shown in different species, including cats [41] and dogs [42]. For example, a single intradermal DNA immunization in ear pinna, given 1 year prior to infection, protected dogs against rabies virus $[42,43]$.

DNA constructs encoding GnRHR driven by different promoters (CMV, EF1 $\alpha$, or CAG) were generated in this study to identify those that support high antigen expression leading to suppression of reproductive functions in immunized mice. Theoretically, EF1 $\alpha$ and CAG promoters can be superior to CMV because they contain components that might prevent promoter methylation and, therefore, be active for extended periods of time [44-46]. Here, GnRHR plasmid driven by EF $1 \alpha$ promoter was found to be the most effective in suppression of testosterone and reduction of fetuses in mouse fertility trials.

To obtain insights on possible mechanisms leading to testosterone suppression and reduced fertility, expression of GnRHR mRNA was quantified in mouse pituitary glands. A significant decrease in GnRHR mRNA was found in the treatment groups, as compared to controls. This could be due to lysis of GnRHR-expressing cells (gonadotropes) in the pituitary gland by GnRHR-specific cytotoxic T lymphocytes or cytotoxic cells (e.g., natural killer cells, macrophages, monocytes) activated by anti-GnRHR antibodies. It is also 


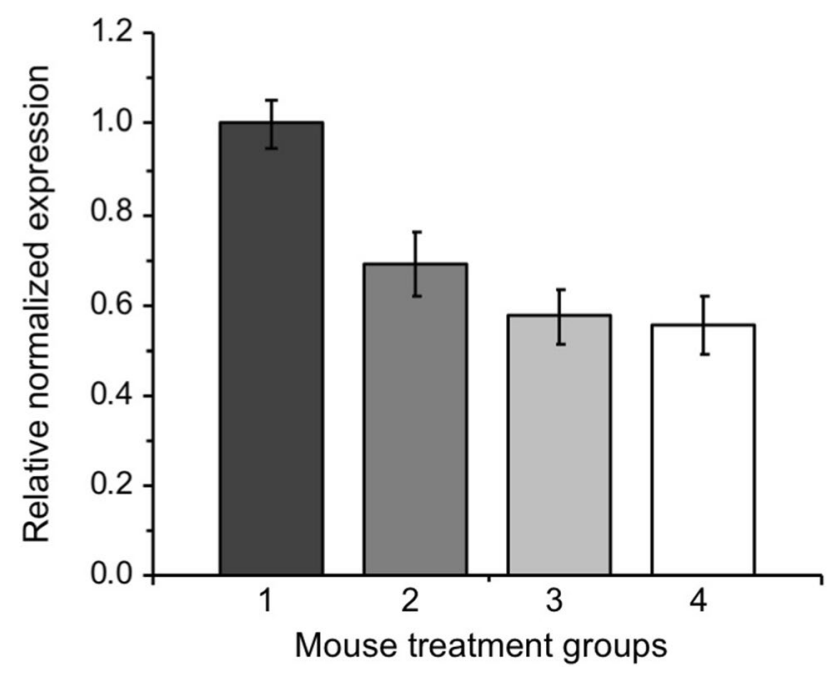

Fig. 5 Quantitative GnRHR mRNA expression in pituitary glands collected from mice immunized with different Ub-fGnRHR constructs. Mouse groups: (1) not treated, (2) immunized with pSF(CMV)-UbfGnRHR construct, (3) immunized with $\mathrm{pSF}(\mathrm{EF} 1 \alpha)$ UbfGnRHR construct, and (4) immunized with pSF(CAG)-UbfGnRHR construct. The pituitary glands were collected at necropsy and total RNA isolated. One-step RT qPCR was performed with iTAq Universal Probes One-Step Kit using CFX96 Real-Time PCR Detection System. Two repeats for each sample were run. The CFX Manager Software incorporated in the equipment calculates relative expression level of the target sequence in treated samples relative to control samples. The data are shown as mean relative normalized expression for each group \pm SEM. The graph data are presented using the "relative to control" option with the $y$ axis scaled from 0 to 1 to quickly visualize up- or downregulation of the target. All treated groups were significant from the control at $p<0.001$

possible that downregulation of GnRHR mRNA was caused by interference of GnRHR antibodies with GnRH-GnRHR binding and/or signaling. Mo et al. [47] demonstrated significant downregulation of GnRHR mRNA in the pituitary after treatment of rats with various $\mathrm{GnRH}$ agonists and antagonists known to influence GnRH-GnRHR interaction. In another example, the amount of GnRHR mRNA in the pituitary was found to be significantly lower in pigs treated with a GnRH-based vaccine designed for immunological castration [48]. Despite the fact that GnRHR mRNA expression was downregulated in all treatment groups in this study, significant suppression of fertility was found only in the group of mice immunized with $\mathrm{pSF}(\mathrm{EF} 1 \alpha)$-UbfGnRHR construct. Additional studies as to cellular localization of the GnRHR protein in gonadotropes of immunized mice will be needed to explain these findings.

While suppression of some reproductive functions in GnRHR-immunized mice was demonstrated, the complete loss of fertility was not achieved, most likely, because expression of the antigen (GnRHR) was not sufficiently high and its duration was not long enough to establish immunological memory. One of the major reasons for transgene

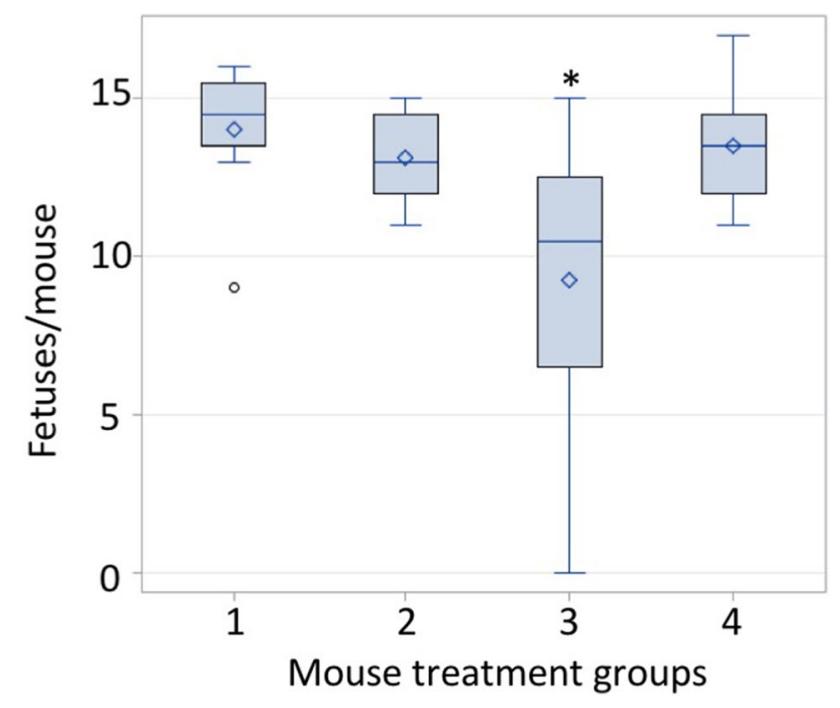

Fig. 6 Fertility trials in mice treated with different Ub-fGnRHR constructs. Groups: (1) control, not treated, (2) immunized with pSF(CMV)-UbfGnRHR construct, (3) immunized with $\operatorname{pSF}(\mathrm{EF} 1 \alpha)$ UbfGnRHR construct, and (4) immunized with pSF(CAG)-UbfGnRHR construct. Number of fetuses per female mouse was counted to compare test groups (immunized males paired with normal females) to the control group (normal males paired with normal females). Significance level of $p<0.05$ is noted with an asterisk

silencing and subsequent loss of expression is $\mathrm{CpG}$ methylation in the promoter area $[49,50]$. To prevent promoter silencing and improve performance of the constructs, the use of CpG-free promoters might be advantageous. Also, DNAGnRHR constructs might be optimized with a dual antigen expression system. Others have shown that a plasmid with a dual antigen expression cassette increased transgene expression significantly as compared to a plasmid with a single cassette [51]. Additional approaches for enhanced and extended GnRHR expression include linearization of the plasmid and minimization of the plasmid bacterial backbone. Linearization of the plasmid allows improved cell transfection. Minimizing bacterial backbone prevents excessive immune reactions against cells transfected with the antigen that might kill the cells [50]. Also, for enhanced antigen expression, delivery of DNA constructs might be combined with electroporation that improves cell transfection drastically [52].

\section{Conclusions}

In this study, we generated DNA-based vaccines directed against a key contraceptive target, the GnRH receptor, something not previously reported. Such DNA constructs were shown to stimulate biologically relevant immune responses against the antigen resulting in suppression of testosterone and impaired fertility in immunized mice. Importantly, this was achieved with a single immunization and without the 
use of adjuvants. The forthcoming research will be focused on generation of improved GnRHR-encoding DNA constructs for induction of immunological memory and subsequent long-lasting loss of fertility in immunized animals. The immunomodulation strategies will also be explored.

Acknowledgements The study was funded by Found Animals Foundation, Michelson Grant in Reproductive Biology D1213-F13 and supported by the Scott-Ritchey Research Center, College of Veterinary Medicine, Auburn University, Auburn, AL, USA.

Open Access This article is distributed under the terms of the Creative Commons Attribution 4.0 International License (http://creativeco mmons.org/licenses/by/4.0/), which permits unrestricted use, distribution, and reproduction in any medium, provided you give appropriate credit to the original author(s) and the source, provide a link to the Creative Commons license, and indicate if changes were made.

\section{References}

1. Strand, P. (2011, November 17) The global stray dog population crisis. Animal Welfare, Canine Issues. http://www.naiaonline.org.

2. Fooks, A. R., Cliquet, F., Finke, S., Freuling, C., Hemachudha, T., Mani, R. S., Müller, T., Nadin-Davis, S., Picard-Meyer, E., Wilde, H., \& Banyard, A. C. (2017). Rabies. Nature Reviews Disease Primers, 30, 17091.

3. Dhillon, J., Hoopes, J., \& Epp, T. (2018). Scoping decades of dog evidence: a scoping review of dog bite-related sequelae. Canadian Journal of Public Health. https://doi.org/10.17269/s4199 7-018-0145-3.

4. Perez-Osorio, C. E., Zavala-Velazquez, J. E., Arias Leon, J. J., \& Zavala-Castro, J. E. (2008). Rickettsia felis as emergent global threat for humans. Emerging Infectious Diseases, 14, 1019-1023.

5. McElroy, K. M., Blagburn, B. L., Breitschwerdt, E. B., Mead, P. S., \& McQuiston, J. H. (2010). Flea-associated zoonotic diseases of cats in the USA: Bartonellosis, flea-borne rickettsioses, and plague. Trends in Parasitology, 26, 197-204.

6. Loss, S. R., Will, T., \& Marra, P. P. (2013). The impact of freeranging domestic cats on wildlife of the United States. Nature Communications, 4, 1396.

7. Fayrer-Hosken, R. (2008). Controlling animal populations using anti-fertility vaccines. Reproduction in Domestic Animals, 43(Suppl 2), 179-185.

8. Kirkpatrick, J. F., Lyda, R. O., \& Frank, K. M. (2011). Contraceptive vaccines for wildlife: A review. American Journal of Reproductive Immunology, 66, 40-50.

9. Levy, J. K. (2011). Contraceptive vaccines for the humane control of community cat populations. American Journal of Reproductive Immunology, 66, 63-70.

10. Swegen, A., \& Aitken, R. J. (2016). Prospects for immunocontraception in feral horse population control: Exploring novel targets for an equine fertility vaccine. Reproduction, Fertility, and Development, 28, 853-863.

11. Gupta, S. K., \& Minhas, V. (2017). Wildlife population management: Are contraceptive vaccines a feasible proposition? Frontiers in Bioscience, 9, 357-374.

12. Kutzler, M. A., \& Weiner, D. B. (2008). DNA vaccines: Ready for prime time? Nature Reviews Genetics, 9, 776-788.

13. Liu, M. A. (2011). DNA vaccines: An historical perspective and view to the future. Immunological Reviews, 239, 62-84.
14. Saade, F., \& Petrovsky, N. (2012). Technologies for enhanced efficacy of DNA vaccines. Expert Review of Vaccines, 11, 189-209.

15. Xiang, R. L., Zhou, F., Yang, Y., \& Peng, J. P. (2003). Construction of the plasmid pCMV4-rZPC' DNA vaccine and analysis of its contraceptive potential. Biology of Reproduction, 68, $1518-1524$.

16. Yu, M. F., Fang, W. N., Xiong, G. F., Yang, Y., \& Peng, J. P. (2011). Evidence for the inhibition of fertilization in vitro by antiZP3 antisera derived from DNA vaccine. Vaccine, 29, 4933-4939.

17. Ma, X., Kadir, Z., Li, J., \& Zhang, F. (2012). The effects of GM$\mathrm{CSF}$ and IL-5 as molecular adjuvants on immune responses and contraception induced by mZP3 DNA vaccination. American Journal of Reproductive Immunology, 68, 476-485.

18. Wang, Y., Zhang, B., Li, J., Aipire, A., Li, Y., \& Zhang, F. (2018). Enhanced contraception of canine zona pellucida 3 DNA vaccine via targeting DEC-205 in mice. Theriogenology., $113,56-62$.

19. Sun, Z., Jin, F., Li, Y., \& Zhang, J. (2010). Immunocontraceptive effect of DNA vaccine targeting fertilin beta in male mice. American Journal of Reproductive Immunology, 63, 282-290.

20. Naz, R. K. (2006). Effect of sperm DNA vaccine on fertility of female mice. Molecular Reproduction and Development, 73, 918-928.

21. Naz, R. K. (2006). Effect of fertilization antigen (FA-1) DNA vaccine on fertility of female mice. Molecular Reproduction and Development, 73, 1473-1479.

22. Khan, M. A., Ferro, V. A., Koyama, S., Kinugasa, Y., Song, M., Ogita, K., Tsutsui, T., Murata, Y., \& Kimura, T. (2007). Immunisation of male mice with a plasmid DNA vaccine encoding gonadotrophin releasing hormone (GnRH-I) and T-helper epitopes suppresses fertility in vivo. Vaccine, 25, 3544-3553.

23. Khan, M. A., Ogita, K., Ferro, V. A., Kumasawa, K., Tsutsui, T., \& Kimura, T. (2008). Immunisation with a plasmid DNA vaccine encoding gonadotrophin releasing hormone (GnRH-I) and T-helper epitopes in saline suppresses rodent fertility. Vaccine, 26, 1365-1374.

24. Liu, T., Huang, J. C., Lu, C. L., Yang, J. L., Hu, Z. Y., Gao, F., \& Liu, Y. X. (2010). Immunization with a DNA vaccine of testis-specific sodium-hydrogen exchanger by oral feeding or nasal instillation reduces fertility in female mice. Fertility and Sterility, 93, 1556-1566.

25. Yu, Q., Mei, X. Q., Ding, X. F., Dong, T. T., Dong, W. W., \& Li, H. G. (2015). Construction of a catsper1 DNA vaccine and its antifertility effect on male mice. PLOS ONE., 10(5), e0127508.

26. Bliss, S. P., Navratil, A. M., Xie, J., \& Roberson, M. S. (2010). GnRH signaling, the gonadotrope and endocrine control of fertility. Frontiers in Neuroendocrinology, 31, 322-340.

27. Samoylov, A., Cochran, A., Schemera, B., Kutzler, M., Donovan, C., Petrenko, V., Bartol, F., \& Samoylova, T. (2015). Humoral immune responses against gonadotropin releasing hormone elicited by immunization with phage-peptide constructs obtained via phage display. Journal of Biotechnology, 216, 20-28.

28. Bergman, P. J., McKnight, J., Novosad, A., Charney, S., Farrelly, J., Craft, D., Wulderk, M., Jeffers, Y., Sadelain, M., Hohenhaus, A. E., Segal, N., Gregor, P., Engelhorn, M., Riviere, I., Houghton, A. N., \& Wolchok, J. D. (2003). Long-term survival of dogs with advanced malignant melanoma after DNA vaccination with xenogeneic human tyrosinase: A phase I trial. Clinical Cancer Research, 9, 1284-1290.

29. Liao, J. C., Gregor, P., Wolchok, J. D., Orlandi, F., Craft, D., Leung, C., Houghton, A. N., \& Bergman, P. J. (2006). Vaccination with human tyrosinase DNA induces antibody responses in dogs with advanced melanoma. Cancer Immunity Archive, 6, 8.

30. Saxena, B. B., Clavio, A., Singh, M., Rathnam, P., Bukharovich, Y., Reimers, T. Jr., Saxena, A., \& Perkins, S. (2002). Modulation of ovarian function in female dogs immunized with bovine 
luteinizing hormone receptor. Reproduction in Domestic Animals, 37, 9-17.

31. Saxena, B. B., Clavio, A., Singh, M., Rathnam, P., Bukharovich, E.Y., Reimers, T. J. Jr., Saxena, A., \& Perkins, S. (2003). Effect of immunization with bovine luteinizing hormone receptor on ovarian function in cats. American Journal of Veterinary Research, 64, 292-298.

32. Remy, J. J., Bozon, V., Couture, L., Goxe, B., Salesse, R., \& Garnier, J. (1993). Suppression of fertility in male mice by immunization against LH receptor. Journal of Reproductive Immunology, 1 , 63-79.

33. Yan, P., He, W., Liang, Z., Chen, Z., Shang, X., He, H., Tang, Y., Ni, B., Zhang, J., Shen, Z., Wu, Y., \& Li, J. (2009). A novel dominant B-cell epitope of FSHR identified by molecular docking induced specific immune response and suppressed fertility. Gynecological Endocrinology, 12, 828-838.

34. Yang, L. H., Li, J. T., Yan, P., Liu, H. L., Zeng, S. Y., Wu, Y. Z., Liang, Z. Q., \& He, W. (2011). Follicle-stimulating hormone receptor (FSHR)-derived peptide vaccine induced infertility in mice without pathological effect on reproductive organs. Reproduction, Fertility, and Development, 23, 544-550.

35. Clague, M. J., Heride, C., \& Urbé, S. (2015). The demographics of the ubiquitin system. Trends in Cell Biology, 25, 417-426.

36. Zhang, M., Obata, C., Hisaeda, H., Ishii, K., Murata, S., Chiba, T., Tanaka, K., Li, Y., Furue, M., Chou, B., Imai, T., Duan, X., \& Himeno, K. (2005). A novel DNA vaccine based on ubiquitinproteasome pathway targeting 'self'-antigens expressed in melanoma/melanocyte. Gene Therapy, 12, 1049-1057.

37. Chen, J. H., Yu, Y. S., Liu, H. H., Chen, X. H., Xi, M., Zang, G. Q., \& Tang, Z. H. (2011). Ubiquitin conjugation of hepatitis $B$ virus core antigen DNA vaccine leads to enhanced cell-mediated immune response in BALB/c mice. Hepatitis Monthly, 11, 620-628.

38. Chou, B., Hiromatsu, K., Okano, S., Ishii, K., Duan, X., Sakai, T., Murata, S., Tanaka, K., \& Himeno, K. (2012). Antiangiogenic tumor therapy by DNA vaccine inducing aquaporin-1-specific CTL based on ubiquitin-proteasome system in mice. The Journal of Immunology, 189, 1618-1626.

39. Förg, P., von Hoegen, P., Dalemans, W., \& Schirrmacher, V. (1998). Superiority of the ear pinna over muscle tissue as site for DNA vaccination. Gene Therapy, 5, 789-797.

40. Jurianz, K., von Hoegen, P., \& Schirrmacher, V. (1998). Superiority of the ear pinna over a subcutaneous tumour inoculation site for induction of a Th1-type cytokine response. Cancer Immunology, Immunotherapy, 45, 327-333.

41. Tesoro-Cruz, E., Calderón-Rodríguez, R., Hernández-González, R., Blanco-Favéla, F., \& Aguilar-Setién, A. (2008). Intradermal DNA vaccination in ear pinnae is an efficient route to protect cats against rabies virus. Veterinary Research, 39, 16.
42. Lodmell, D. L., Parnell, M. J., Weyhrich, J. T., \& Ewalt, L. C. (2003). Canine rabies DNA vaccination: A single-dose intradermal injection into ear pinnae elicits elevated and persistent levels of neutralizing antibody. Vaccine, 21, 3998-4002.

43. Lodmell, D. L., Ewalt, L.,C., Parnell, M. J., Rupprecht, C. E., \& Hanlon, C. A. (2006). One-time intradermal DNA vaccination in ear pinnae one year prior to infection protects dogs against rabies virus. Vaccine, 24, 412-416.

44. Garg, S., Oran, A. E., Hon, H., \& Jacob, J. (2004). The hybrid cytomegalovirus enhancer/chicken beta-actin promoter along with woodchuck hepatitis virus posttranscriptional regulatory element enhances the protective efficacy of DNA vaccines. The Journal of Immunology, 173, 550-558.

45. Qin, J. Y., Zhang, L., Clift, K. L., Hulur, I., Xiang, A. P., Ren, B. Z., \& Lahn, B. T. (2010) Systematic comparison of constitutive promoters and the doxycycline-inducible promoter. PLOS ONE, 5, e10611.

46. Hazen, M., Bhakta, S., Vij, R., Randle, S., Kallop, D., Chiang, V., Hötzel, I., Jaiswal, B. S., Ervin, K. E., Li, B., Weimer, R. M., Polakis, P., Scheller, R. H., Junutula, J. R., \& Hongo, J. A. (2014) An improved and robust DNA immunization method to develop antibodies against extracellular loops of multi-transmembrane proteins. MAbs 6, 95-107.

47. Mo, Y., Peng, P., Zhou, R., He, Z., Huang, L., \& Yang, D. (2010). Regulation of gonadotropin-releasing hormone $(\mathrm{GnRH})$ receptorI expression in the pituitary and ovary by a GnRH agonist and antagonist. Reproductive Sciences, 17, 68-77.

48. Fang, F., Li, H., Liu, Y., Zhang, Y., Tao, Y., Li, Y., Cao, H., Wang, S., Wang, L., \& Zhang, X. (2010). Active immunization with recombinant $\mathrm{GnRH}$ fusion protein in boars reduces both testicular development and mRNA expression levels of GnRH receptor in pituitary. Animal Reproduction Science, 119, 275-281.

49. Papagatsias, T., Rozis, G., Athanasopoulos, T., Gotch, F., Dickson, G., \& Patterson, S. (2008). Activity of different vaccine-associated promoter elements in human dendritic cells. Immunology Letters, 115, 117-125.

50. Wong, S. P., Argyros, O., \& Harbottle, R. P. (2015). Sustained expression from DNA vectors. Advances in Genetics, 89, $113-152$.

51. Sasaki, S., Takeshita, F., Oikawa, T., Kojima, Y., Xin, K. Q., Okuda, K., \& Ishii, N. (2004). Improvement of DNA vaccine immunogenicity by a dual antigen expression system. Biochemical and Biophysical Research Communications, 315, 38-43.

52. Sardesai, N. Y., \& Weiner, D. B. (2011). Electroporation delivery of DNA vaccines: Prospects for success. Current Opinion in Immunology, 23, 421-429. 